\title{
The number of plane conics that are five-fold tangent to a given curve
}

\author{
Andreas Gathmann
}

\begin{abstract}
Given a general plane curve $Y$ of degree $d$, we compute the number $n_{d}$ of irreducible plane conics that are five-fold tangent to $Y$. This problem has been studied before by Vainsencher using classical methods, but it could not be solved because the calculations produced too many non-enumerative correction terms that could not be analyzed. In our current approach, we express the number $n_{d}$ in terms of relative Gromov-Witten invariants that can then be directly computed. As an application, we consider the K3 surface given as the double cover of $\mathbb{P}^{2}$ branched along a sextic curve. We compute the number of rational curves in this K3 surface in the homology class that is the pull-back of conics in $\mathbb{P}^{2}$, and compare this number with the corresponding Yau-Zaslow K3 invariant. This gives an example of such a K3 invariant for a non-primitive homology class.
\end{abstract}

Let $Y \subset \mathbb{P}^{2}$ be a generic plane curve of degree $d \geqslant 5$. We want to consider smooth plane conics that are five-fold tangent to $Y$. As the space of all plane conics is five-dimensional and each tangency imposes one condition on the curves, we expect a finite number of such five-fold tangent conics. It will be easy to see that this number is indeed finite; let us call it $n_{d}$. The goal of this paper is to compute it.

Of course this is a classical problem, and attempts have been made to solve it using classical methods of enumerative geometry. Vainsencher [Vai98] tried to use various blow-ups of the ordinary $\mathbb{P}^{5}$ of conics as moduli spaces, but the intersection of the five tangency conditions in this moduli space always resulted in a scheme with many non-enumerative and non-reduced components whose geometry was so complicated that the problem could not be solved that way.

In this paper we use different moduli spaces, namely moduli spaces of relative stable maps, to solve the problem. There is a well-defined compact moduli space $\bar{M}_{(2,2,2,2,2)}^{Y}\left(\mathbb{P}^{2}, 2\right) \subset \bar{M}_{0,5}\left(\mathbb{P}^{2}, 2\right)$ that parametrizes rational stable maps to $\mathbb{P}^{2}$ of degree 2 (i.e. conics) with five marked points such that the stable map is tangent to $Y$ at all of these points. It comes equipped with a zero-dimensional virtual fundamental class, whose degree $N_{d}$ can be computed explicitly using the methods of [Gat02].

We can interpret the number $N_{d}$ as the 'virtual number' of conics that are five-fold tangent to $Y$. It is only virtual because it contains, just as in Vainsencher's classical computations, nonenumerative contributions from the 'boundary' of the moduli space. These contributions are quite simple however. It is not hard to see that the only degree-2 rational stable maps $f: C \rightarrow \mathbb{P}^{2}$ that satisfy the tangency conditions at the five marked points are all double covers of a bitangent of $Y$, and have the marked points distributed in one of the following two ways:

Received 5 August 2003, accepted in final form 22 March 2004, published online 10 February 2005. 2000 Mathematics Subject Classification 14N35.

Keywords: enumerative geometry, relative Gromov-Witten invariants, K3 surfaces.

Funded by the NSF grant DMS 9729992.

This journal is (C) Foundation Compositio Mathematica 2005. 


\section{A. Gathmann}

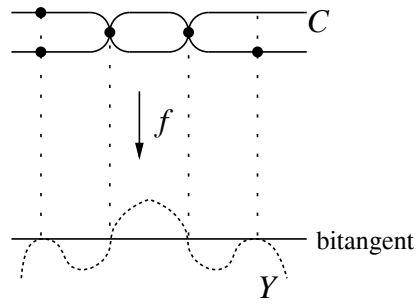

(a)

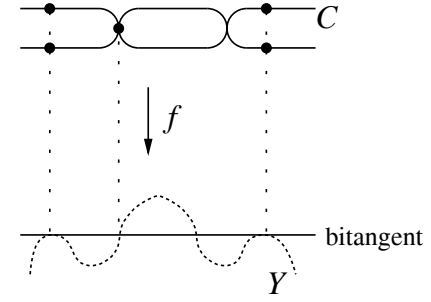

(b)

FIGURE 1.

There are only finitely many stable maps with marked points as in Figure 1(a), so we just have to count them and subtract their number from the virtual invariant $N_{d}$. However, Figure 1(b) shows a one-dimensional family of stable maps (the unmarked ramification point of $f$ can move). We use equations from relative Gromov-Witten theory to compute the degree of the zero-dimensional virtual fundamental class of the moduli space on this one-dimensional component. By subtracting both correction terms, we finally arrive at the enumerative numbers $n_{d}$. They are

$$
n_{d}=\frac{1}{5 !} d(d-3)(d-4)\left(d^{7}+12 d^{6}-18 d^{5}-540 d^{4}+251 d^{3}+5712 d^{2}-1458 d-14580\right) .
$$

Our (as well as Vainsencher's) motivation for studying this problem came from a question concerning rational curves in $\mathrm{K} 3$ surfaces. If $X$ is a $\mathrm{K} 3$ surface and $\beta \in H_{2}(X, \mathbb{Z})$ the homology class of a holomorphic curve in $X$, then various authors [YZ96, Bea99, Got98, BL00] have shown that the number of rational curves in $X$ of class $\beta$ is equal to the $q^{d}$ coefficient of the series

$$
G(q)=\prod_{i>0} \frac{1}{\left(1-q^{i}\right)^{24}}=1+24 q+324 q^{2}+3200 q^{3}+25650 q^{4}+176256 q^{5}+\cdots
$$

with $d=\frac{1}{2} \beta^{2}+1$, if the class $\beta$ is primitive, i.e. not a non-trivial multiple of a smaller integral homology class. There is also a well-defined 'K3 invariant' (using a modified obstruction theory on the moduli spaces of stable maps to $X$ ) for non-primitive $\beta$ (see [BL00]); however, it is not yet known how this invariant relates to the above series $G(q)$ or to the enumerative number.

The result of this paper allows us to study this question in a non-trivial example: we let $X$ be the double cover of $\mathbb{P}^{2}$ branched along a sextic curve $Y$, and take $\beta$ to be the pull-back of the class of conics in $\mathbb{P}^{2}$. Our work allows us to compute the enumerative number of rational curves in $X$ of class $\beta$, which we can then compare with the corresponding number of the series $G(q)$. The result is that the ' $\mathrm{K} 3$ invariant' is equal to the corresponding term in the $G(q)$ series, plus a double cover correction term that is equal to $\frac{1}{8}$ times the number of rational curves in $X$ of class $\frac{1}{2} \beta$. Note that this is the same sort of correction term as for multiple covers of rational curves in Calabi-Yau threefolds. We conjecture that this pattern continues for classes $\beta$ of higher divisibility.

The paper is organized as follows. In $\S 1$ we show how to compute the relative Gromov-Witten invariant $N_{d}$. We analyze the moduli space $\bar{M}_{(2,2,2,2,2)}^{Y}\left(\mathbb{P}^{2}, 2\right)$ and its virtual fundamental class in $\S \S 2$ and 3, respectively, leading to the final result for $n_{d}$ in Corollary 3.6. Section 4 contains the application to K3 surfaces mentioned above.

\section{Relative Gromov-Witten invariants}

In this section we show how to compute the relative Gromov-Witten invariant that corresponds to the number of conics that are five-fold tangent to a given smooth plane curve. We use the notation and results from [Gat02], to which we also refer for further details. 


\section{The NUMBer of PlANE CONICS THAT ARE FIVE-FOLD TANGENT TO A GIVEN CURVE}

Definition 1.1. Let $Y \subset \mathbb{P}^{2}$ be a smooth curve of degree $d$, and let $m_{1}, \ldots, m_{n}$ be non-negative integers. We denote by $\bar{M}_{\left(m_{1}, \ldots, m_{n}\right)}=\bar{M}_{\left(m_{1}, \ldots, m_{n}\right)}^{Y}\left(\mathbb{P}^{2}, 2\right)$ the moduli space of $n$-pointed relative stable maps of degree 2 to $\mathbb{P}^{2}$ relative to $Y$ with multiplicities $m_{1}, \ldots, m_{n}$, as defined in [Gat02, Definitions 1.1 and 1.18]. Its virtual fundamental class is denoted by $\left[\bar{M}_{\left(m_{1}, \ldots, m_{n}\right)}\right]^{\text {virt }}$.

Remark 1.2. The moduli space $\bar{M}_{\left(m_{1}, \ldots, m_{n}\right)}$ can be thought of as a compactification of the space of irreducible plane conics together with $n$ distinct marked points on them at which the conic has the prescribed local intersection multiplicities $m_{1}, \ldots, m_{n}$ with $Y$. In particular, the moduli space $\bar{M}_{(2,2,2,2,2)}$ corresponds to conics that are five-fold tangent to $Y$.

Remark 1.3. For future reference let us recall the precise definition from [Gat02]. Consider the degree- $d$ Veronese embedding $i: \mathbb{P}^{2} \rightarrow \mathbb{P}^{D}$ with $D=\left(\begin{array}{c}d+2 \\ 2\end{array}\right)-1$. We have $i(Y)=i\left(\mathbb{P}^{2}\right) \cap H$ in $\mathbb{P}^{D}$ for a suitable hyperplane $H \subset \mathbb{P}^{D}$. The inclusion morphism $i$ induces an inclusion of moduli spaces $\bar{M}_{0, n}\left(\mathbb{P}^{2}, 2\right) \subset \bar{M}_{0, n}\left(\mathbb{P}^{D}, 2 d\right)$. Moreover, let $\bar{M}_{\left(m_{1}, \ldots, m_{n}\right)}^{H}\left(\mathbb{P}^{D}, 2 d\right)$ be the closure in $\bar{M}_{0, n}\left(\mathbb{P}^{D}, 2 d\right)$ of all stable maps $\left(C, x_{1}, \ldots, x_{n}, f\right)$ such that $C$ is irreducible, $f(C) \not \subset H$, and the divisor $f^{*} Y$ on $C$ contains the points $x_{i}$ with multiplicities $m_{i}$. Then we define

$$
\bar{M}_{\left(m_{1}, \ldots, m_{n}\right)}:=\bar{M}_{0, n}\left(\mathbb{P}^{2}, 2\right) \cap \bar{M}_{\left(m_{1}, \ldots, m_{n}\right)}^{H}\left(\mathbb{P}^{D}, 2 d\right)
$$

(with the intersection taken in $\bar{M}_{0, n}\left(\mathbb{P}^{D}, 2 d\right)$ ). The virtual fundamental class is the corresponding refined intersection product

$$
\left[\bar{M}_{\left(m_{1}, \ldots, m_{n}\right)}\right]^{\mathrm{virt}}:=\left[\bar{M}_{0, n}\left(\mathbb{P}^{2}, 2\right)\right] \cdot\left[\bar{M}_{\left(m_{1}, \ldots, m_{n}\right)}^{H}\left(\mathbb{P}^{D}, 2 d\right)\right] \in A_{*}\left(\bar{M}_{\left(m_{1}, \ldots, m_{n}\right)}\right) .
$$

The virtual dimension of $\bar{M}_{\left(m_{1}, \ldots, m_{n}\right)}$ is $5-\sum_{i}\left(m_{i}-1\right)$. By abuse of notation, we always drop the superscript virt from the notation of the virtual fundamental class from now on, as we do not need the ordinary fundamental classes of these spaces.

Remark 1.4. From Remark 1.3 we immediately obtain the following statement: let $\mathcal{C}=\left(C, x_{1}, \ldots\right.$, $\left.x_{n}, f\right) \in \bar{M}_{\left(m_{1}, \ldots, m_{n}\right)}$ be an automorphism-free stable map such that $C \cong \mathbb{P}^{1}$ is irreducible and $f(C) \not \subset Y$. Then, locally around this point, $\bar{M}_{\left(m_{1}, \ldots, m_{n}\right)}$ is scheme-theoretically the subscheme of $\bar{M}_{0, n}\left(\mathbb{P}^{2}, 2\right)$ given by the $\sum_{i} m_{i}$ equations that describe the vanishing of the $m_{i}$-jets of $e v_{i}^{*} Y$ at the points $x_{i}$, where $e v_{i}: \bar{M}_{0, n}\left(\mathbb{P}^{2}, 2\right) \rightarrow \mathbb{P}^{2}$ are the evaluation maps. Moreover, if $\bar{M}_{\left(m_{1}, \ldots, m_{n}\right)}$ has the expected dimension at this point $\mathcal{C}$, then this point lies on a unique irreducible component of $\bar{M}_{\left(m_{1}, \ldots, m_{n}\right)}$, and the virtual fundamental class on this component is just the ordinary schemetheoretic fundamental class, i.e. the length of the scheme $\bar{M}_{\left(m_{1}, \ldots, m_{n}\right)}$ at this irreducible component.

Remark 1.5. There is an easier description of $\bar{M}_{\left(m_{1}, \ldots, m_{n}\right)}$ as a set. Namely, $\bar{M}_{\left(m_{1}, \ldots, m_{n}\right)}$ is the subspace of $\bar{M}_{0, n}\left(\mathbb{P}^{2}, 2\right)$ of all $n$-pointed rational stable maps $\left(C, x_{1}, \ldots, x_{n}, f\right)$ of degree 2 to $\mathbb{P}^{2}$ such that the following two conditions are satisfied:

(i) $f\left(x_{i}\right) \in Y$ for all $i$ such that $m_{i}>0$;

(ii) $f^{*} Y-\sum_{i} m_{i} x_{i} \in A_{0}\left(f^{-1}(Y)\right)$ is effective.

As mentioned above, the moduli space $\bar{M}_{(2,2,2,2,2)}$ has virtual dimension zero and corresponds to conics five-fold tangent to $Y$ (together with a labeling of the five tangency points). Hence, we define the following.

DeFinition 1.6. The number

$$
N_{d}:=\frac{1}{5 !} \cdot \operatorname{deg}\left[\bar{M}_{(2,2,2,2,2)}\right] \in \mathbb{Q}
$$

will be called the virtual number of conics five-fold tangent to $Y$. 


\section{A. Gathmann}

The number $N_{d}$ is only virtual because it receives correction terms from double covers of lines (see $\S \S 2$ and 3 ). In the rest of this section we show how to compute the number $N_{d}$. Obviously, we can assume that $d \geqslant 5$.

The computation is done using the main Theorem 2.6 of [Gat02] that tells us 'how to raise the multiplicities of the moduli spaces': it states that

$$
\left(e v_{n}^{*} Y+m_{n} \psi_{n}\right) \cdot\left[\bar{M}_{\left(m_{1}, \ldots, m_{n}\right)}\right]=\left[\bar{M}_{\left(m_{1}, \ldots, m_{n-1}, m_{n}+1\right)}\right]+\text { correction terms, }
$$

where $e v_{n}: \bar{M}_{\left(m_{1}, \ldots, m_{n}\right)} \rightarrow \mathbb{P}^{2}$ is the evaluation map at the last marked point, and $\psi_{n}$ is the first Chern class of the cotangent line bundle $L_{n}$, i.e. of the bundle whose fiber at a stable map $\left(C, x_{1}, \ldots, x_{n}, f\right)$ is the cotangent space $T_{C, x_{n}}^{\vee}$. The correction terms are as follows. Every correction term corresponds to a moduli space of reducible curves with $r+1$ components $C_{0}, \ldots, C_{r}$, where $C_{0}$ is contracted ${ }^{1}$ to a point of $Y$, and the other components $C_{i}$ intersect $C_{0}$ in a point where they have local intersection multiplicity $\mu_{i}$ to $Y$. We get such a correction term for every $r$, every choice of $\mu_{i}$, and every splitting of the total homology class and the marked points onto the components $C_{i}$, such that the following two conditions are satisfied:

(a) the last marked point $x_{n}$ lies on the component $C_{0}$;

(b) the sum of all $\mu_{i}$ is equal to the sum of those $m_{i}$ for which $x_{i} \in C_{0}$.

These correction terms appear in the above equation with multiplicity $\prod_{i=1}^{r} \mu_{i}$.

EXAmPle 1.7. Here is an example of Equation (1). In the case

$$
\left(e v_{5}^{*} Y+\psi_{5}\right) \cdot\left[\bar{M}_{(2,2,2,2,1)}\right]=\left[\bar{M}_{(2,2,2,2,2)}\right]+\text { correction terms }
$$

we want to figure out the correction terms. As this is an equation in (virtual) dimension 0 , the contracted component $C_{0}$ must have exactly three special points (it would not be stable if it had fewer, and it would have moduli if it had more). Hence, the correction terms fall into the following two categories:

(i) $r=1$ (in the above notation), $C_{1}$ is a conic, and $C_{0}$ is a contracted component with three special points $x_{5}$, the intersection point with $C_{1}$, and one other $x_{i}$ for $i=1, \ldots, 4$;

(ii) $r=2, C_{1}$ and $C_{2}$ are two lines, and $C_{0}$ is a contracted component with three special points $x_{5}$ and the two intersection points with $C_{1}$ and $C_{2}$.

Actually, case (ii) cannot occur, because condition (b) above cannot be satisfied: the sum $\mu_{1}+\mu_{2}$ is at least 2 , whereas $m_{5}$ is only 1 . Hence, the only correction terms are of type (i). We get four of them: one for each choice of the point $x_{i}$ that is to lie on the contracted component $C_{0}$. We have $\mu_{1}=m_{i}+m_{5}=3$ in each of these cases by condition (b). All four correction terms appear with multiplicity $\mu_{1}=3$. Pictorially, the equation reads

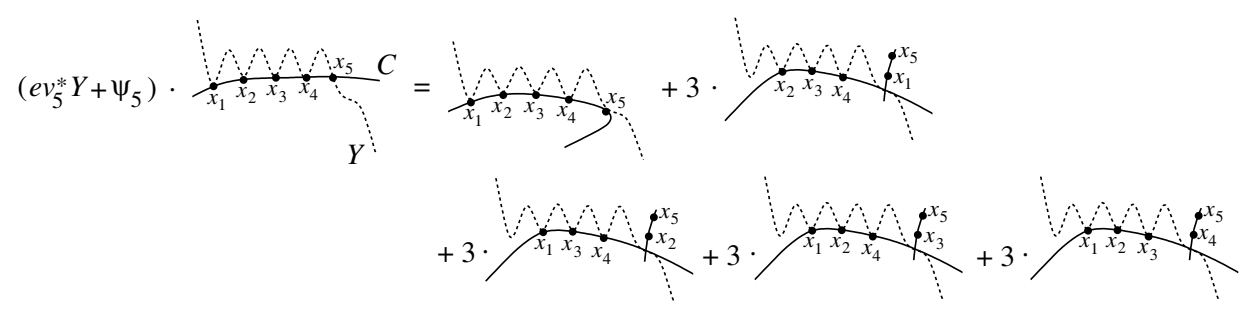

Here, the dotted curve is the fixed curve $Y$, and the full curve is the moving conic $C$. In the four correction terms, the component with $x_{5}$ on it is meant to be contracted. Written down as an

\footnotetext{
${ }^{1}$ This uses the fact that the curve $Y$ has positive genus, and that therefore every rational stable map to $Y$ must be constant. In general, $C_{0}$ can be a curve with any homology class in $Y$.
} 


\section{The NUMBer of PlANE CONICS THAT ARE FIVE-FOLD TANGENT TO A GIVEN CURVE}

equation of virtual fundamental classes of moduli spaces, the formula reads

$$
\left(e v_{5}^{*} Y+\psi_{5}\right) \cdot\left[\bar{M}_{(2,2,2,2,1)}\right]=\left[\bar{M}_{(2,2,2,2,2)}\right]+3\left[\bar{M}_{(3,2,2,2)}\right]+3\left[\bar{M}_{(2,3,2,2)}\right]+3\left[\bar{M}_{(2,2,3,2)}\right]+3\left[\bar{M}_{(2,2,2,3)}\right] .
$$

Remark 1.8. The correction terms in Equation (1) are themselves products of moduli spaces of relative stable maps of the form $\bar{M}_{\left(m_{1}^{\prime}, \ldots, m_{k}^{\prime}\right)}^{Y}\left(\mathbb{P}^{2}, d^{\prime}\right)$ with $d^{\prime} \leqslant 2$ and $m_{1}^{\prime}+\cdots m_{k}^{\prime} \leqslant m_{1}+\cdots m_{n}$. In other words, this equation expresses invariants (i.e. intersection products of $e v_{i}^{*} H$ and $\psi_{i}$ classes, where $H$ is a line in $\left.\mathbb{P}^{2}\right)$ on the relative moduli space $\bar{M}_{\left(m_{1}, \ldots, m_{n}+1\right)}$ in terms of other invariants on relative moduli spaces $\bar{M}_{\left(m_{1}^{\prime}, \ldots, m_{k}^{\prime}\right)}^{Y}\left(\mathbb{P}^{2}, d^{\prime}\right)$ whose 'total multiplicity' $\sum_{i} m_{i}^{\prime}$ is smaller than the total multiplicity $1+\sum_{i} m_{i}$ of $M_{\left(m_{1}, \ldots, m_{n}+1\right)}$. Hence, applying Equation (1) recursively $m_{1}+\cdots+m_{n}$ times, we can express every invariant on $\bar{M}_{\left(m_{1}, \ldots, m_{n}\right)}$ in terms of invariants on $\bar{M}_{(0, \ldots, 0)}$, which are just ordinary Gromov-Witten invariants of $\mathbb{P}^{2}$. As the Gromov-Witten invariants of $\mathbb{P}^{2}$ are wellknown, we can thus compute all relative Gromov-Witten invariants recursively, in particular $N_{d}$. Example 1.7 is the first step in this recursion process; it expresses the invariant $\bar{M}_{(2,2,2,2,2)}$ (with total multiplicity 10) in terms of invariants with total multiplicity 9.

Without actually carrying out the recursion, we can see the following.

Lemma 1.9. The function $d \mapsto N_{d}$ is a polynomial of degree 10 with leading coefficient $1 / 5$ !.

Proof. Using Equation (1) it is easy to show by induction that every invariant (i.e. intersection product of $e v_{i}^{*} H$ and $\psi_{i}$ classes) on a moduli space $\bar{M}_{\left(m_{1}, \ldots, m_{n}\right)}$ is a polynomial in $d$ of degree (at most) $m_{1}+\cdots+m_{n}$. In fact, this is obvious for $m_{1}+\cdots+m_{n}=0$, as we then just have ordinary Gromov-Witten invariants of $\mathbb{P}^{2}$ (that do not depend on $Y$ ). Equation (1) reads

$$
\left[\bar{M}_{\left(m_{1}, \ldots, m_{n-1}, m_{n}+1\right)}\right]=\left(d e v_{n}^{*} H+m_{n} \psi_{n}\right) \cdot\left[\bar{M}_{\left(m_{1}, \ldots, m_{n}\right)}\right]-\text { (correction terms). }
$$

All correction terms have total multiplicity at most $m_{1}+\cdots+m_{n}$, so by the induction hypothesis they contribute a polynomial in $d$ of degree at most $m_{1}+\cdots+m_{n}$. The same is true for the $\psi_{n}$ summand on the right-hand side. Hence, as every invariant on $\bar{M}_{\left(m_{1}, \ldots, m_{n}\right)}$ is a polynomial in $d$ of degree at most $m_{1}+\cdots+m_{n}$ by assumption, it follows that every invariant on $\bar{M}_{\left(m_{1}, \ldots, m_{n}+1\right)}$ is a polynomial in $d$ of degree at most $m_{1}+\cdots+m_{n}+1$.

It can be seen from the same recursive formula that the $d^{10}$ coefficient of the invariant $\operatorname{deg}\left[\bar{M}_{(2,2,2,2,2)}\right]$ is just

$$
\prod_{i=1}^{5} e v_{i}^{*} H^{2} \cdot\left[\bar{M}_{0,5}\left(\mathbb{P}^{2}, 2\right)\right],
$$

i.e. the number of conics through five general points in the plane. This number is 1, proving the statement of the lemma about the leading coefficient.

The precise form of the polynomial $N_{d}$ is quite complicated and can only be obtained by carrying out the full recursion as described above. We only give the result here; a Maple program to compute it can be obtained from the author on request.

Proposition 1.10. For $d \geqslant 5$, the virtual number of conics five-fold tangent to $Y$ is

$$
N_{d}=\frac{1}{5 !} d(d-3)(d-4)\left(d^{7}+12 d^{6}-18 d^{5}-540 d^{4}+311 d^{3}+5457 d^{2}-2133 d-12690\right) .
$$

Remark 1.11. The first few values of $N_{d}$ are given in the following table.

\begin{tabular}{|c|c|c|c|c|c|c|}
\hline$d$ & 5 & 6 & 7 & 8 & 9 & 10 \\
\hline$N_{d}$ & 1985 & 71442 & 687897 & 3893256 & 16180398 & 54679380 \\
\hline
\end{tabular}




\section{A. Gathmann}

Remark 1.12. In this section we have only used Equation (1) in the Chow ring of the moduli space of stable maps $\bar{M}_{0, n}\left(\mathbb{P}^{2}, 2\right)$. In fact, there is a refined version of this equation that we need in $\S 3$. If $\mathcal{P}_{k}$ denotes the rank- $(k+1)$ bundle of (relative) $k$-jets of $e v_{n}^{*} \mathcal{O}(Y)$, there is a section $\sigma$ of the line bundle $\mathcal{P}_{m_{n}} / \mathcal{P}_{m_{n}-1}$ on $\bar{M}_{\left(m_{1}, \ldots, m_{n}\right)}$ whose vanishing precisely describes the condition that the map $f$ of a stable map $\left(C, x_{1}, \ldots, x_{n}, f\right)$ has multiplicity (at least) $m_{n}+1$ to $Y$ at $x_{n}$. The first Chern class of this line bundle is $e v_{n}^{*} Y+m_{n} \psi_{n}$. (In fact, this is the idea behind the proof of (1).) Equation (1) then generalizes to hold also in the Chow group of the zero locus of the section $\sigma$ on $\bar{M}_{\left(m_{1}, \ldots, m_{n}\right)}$.

\section{The components of $\bar{M}_{(2,2,2,2,2)}$}

Having just computed the invariant $N_{d}$, we now study its enumerative significance. To do this, we have to identify the components of $\bar{M}_{(2,2,2,2,2)}$ and compute their virtual fundamental classes. We assume from now on that $Y$ is generic of degree $d \geqslant 5$. The equation of $Y$ is $F=\sum_{I} a_{I} z^{I}=0$, where $I$ runs over all multi-indices $\left(i_{0}, i_{1}, i_{2}\right)$ with $i_{0}+i_{1}+i_{2}=d$, and $z_{0}, z_{1}, z_{2}$ are the homogeneous coordinates on $\mathbb{P}^{2}$. We start with irreducible stable maps whose image is a smooth conic.

Lemma 2.1. Let $m_{1}, \ldots, m_{n}$ be non-negative integers such that $\sum m_{i} \leqslant 10$, and let $\mathcal{C} \in \bar{M}_{\left(m_{1}, \ldots, m_{n}\right)}$ be an irreducible stable map that is not a double cover of a line. Then:

(i) the moduli space $\bar{M}_{\left(m_{1}, \ldots, m_{n}\right)}$ is smooth of dimension $5-\sum_{i}\left(m_{i}-1\right)$ at $\mathcal{C}$ (which is the expected dimension);

(ii) if this expected dimension is negative, then there is no such point $\mathcal{C}$.

Proof. The plane degree- $d$ curves are parametrized by a projective space $\mathbb{P}^{D}$ with $D=\frac{1}{2} d(d+3)$, whose coordinates are the coefficients $a_{I}$ of $F$. Let $Z \subset \bar{M}_{0, n}\left(\mathbb{P}^{2}, 2\right) \times \mathbb{P}^{D}$ be the closed substack of pairs $\left(\left(C, x_{1}, \ldots, x_{n}, f\right), Y\right)$ such that the pull-back by $f$ of the equation of $Y$ vanishes at the points $x_{i}$ to order $m_{i}$ for all $i$. We claim that $Z$ is smooth of the expected dimension at every point $\left(\left(C, x_{1}, \ldots, x_{n}, f\right), Y\right)$ such that $C$ is irreducible and $f$ is not a double cover of a line.

To prove this, we have to show that the matrix of derivatives of the equations describing $Z$ has maximal rank at the given point $\left(\left(C, x_{1}, \ldots, x_{n}, f\right), Y\right)$. By a projective coordinate transformation of $\mathbb{P}^{2}$ and choosing homogeneous coordinates on $C \cong \mathbb{P}^{1}$, we can assume that the map $f$ is given by $(s: t) \mapsto\left(s^{2}: s t: t^{2}\right)$, and the $n$ marked points are $\left(1: \lambda_{i}\right)$ with pairwise distinct $\lambda_{i}$.

Let us now write down the derivatives of the multiplicity equations with respect to the first $m:=\sum_{i} m_{i}$ of the variables $a_{(d, 0,0)}, a_{(d-1,1,0)}, a_{(d-1,0,1)}, a_{(d-2,1,1)}, a_{(d-2,0,2)}, a_{(d-3,1,2)}, a_{(d-3,0,3)}$, $a_{(d-4,1,3)}, a_{(d-4,0,4)}, a_{(d-5,1,4)}$ (remember that $m \leqslant 10$ and $\left.d \geqslant 5\right)$. These coordinates are chosen to be the coefficients of $s^{2 d-i} t^{i}$ for $i=0, \ldots, 9$ when we substitute the map $f$ into $F$.

Multiplicity $m_{i}$ at the point $(s: t)=\left(1: \lambda_{i}\right)$ means that $\left.F\right|_{s=1, t=\lambda_{i}+\epsilon}$ has no $\epsilon$ terms of order less than $m_{i}$. So the rows of the derivative matrix are just $\left(\left(\begin{array}{l}i \\ k\end{array}\right) \lambda_{j}^{i-k}\right)_{i=0, \ldots, m-1}$, for $0 \leqslant k<m_{j}$ and $1 \leqslant j \leqslant n$. For example, for $m_{1}=m_{2}=m_{3}=m_{4}=m_{5}=2$ we get the matrix

$$
\left(\begin{array}{ccccc}
1 & \lambda_{1} & \lambda_{1}^{2} & \cdots & \lambda_{1}^{9} \\
0 & 1 & 2 \lambda_{1} & \cdots & 9 \lambda_{1}^{8} \\
\vdots & \vdots & \vdots & \vdots & \vdots \\
1 & \lambda_{5} & \lambda_{5}^{2} & \cdots & \lambda_{5}^{9} \\
0 & 1 & 2 \lambda_{5} & \cdots & 9 \lambda_{5}^{8}
\end{array}\right) .
$$

By subtracting $\lambda_{1}$ times the $i$ th column from the $(i+1)$ th column for $1 \leqslant i<m$ and using induction, we see that the determinant is $\prod_{i<j}\left(\lambda_{i}-\lambda_{j}\right)^{m_{i} m_{j}}$. In particular, it is not zero, so $Z$ is smooth of the expected dimension at $\left(\left(C, x_{1}, \ldots, x_{n}, f\right), Y\right)$. 
The number of Plane CONiCs that ARE Five-Fold tangent to A Given CURVE

(B)

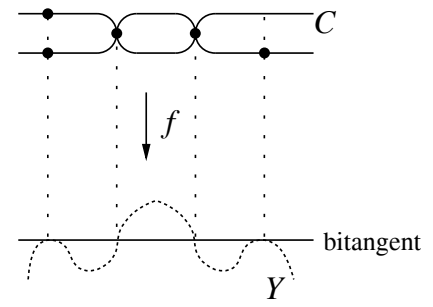

(C)

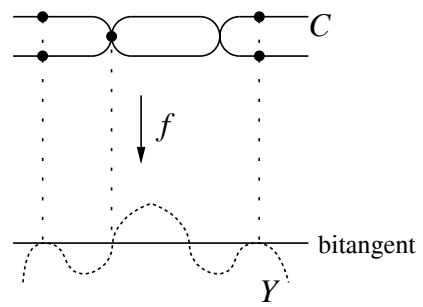

FIGURE 2.

By Remark 1.4, the statement of the lemma is just that the fiber of $Z$ over a general point of $\mathbb{P}^{D}$ is smooth of the expected dimension around a point considered above. This now follows from the Bertini theorem.

Using Remark 1.4 again, the following two corollaries are immediate.

Corollary 2.2. Every irreducible stable map in $\bar{M}_{\left(m_{1}, \ldots, m_{n}\right)}$ whose image in $\mathbb{P}^{2}$ is a smooth conic lies in a unique irreducible component of $\bar{M}_{\left(m_{1}, \ldots, m_{n}\right)}$ of the expected dimension. The virtual fundamental class of this component is equal to the usual one.

Corollary 2.3. The number of smooth plane conics five-fold tangent to $Y$ is finite. We denote it by $n_{d}$.

Now, we study the additional non-enumerative contributions to the virtual invariant $N_{d}$.

Lemma 2.4. The moduli space $\bar{M}_{(2,2,2,2,2)}$ has the following connected components.

(A) 5 ! points for every smooth conic five-fold tangent to $Y$.

(B) $5 ! \cdot(d-4)(d-5)$ points for every bitangent of $Y$, corresponding to double covers of the bitangent, with marked points as in Figure 2(B), i.e. the map is ramified over two transverse intersection points of the bitangent with $Y$, and the five marked points are the two ramification points, both inverse image points of one bitangency point, and one inverse image point of the other bitangency point.

(C) $\frac{1}{4} \cdot 5 ! \cdot(d-4)$ smooth rational curves for every bitangent of $Y$, corresponding to double covers of the bitangent, with marked points as in Figure 2(C), i.e. (for a general stable map in this smooth rational curve) the map is ramified over one transverse intersection point of the bitangent with $Y$ and one other arbitrary point, and the five marked points are the first ramification point and the four inverse image points of the two bitangency points.

Proof. Case 1: the image of the stable map is a smooth conic. If the five marked points are distinct in $\mathbb{P}^{2}$, we get the components $(\mathrm{A})$ by Lemma 2.1 , with the 5 ! corresponding to the labeling of the marked points. If two of the points coincide in $\mathbb{P}^{2}$ (i.e. lie on a contracted component of the stable map), then by the description of $\bar{M}_{(2,2,2,2,2)}$ in Remark 1.5 the conic must have contact of order (at least) 4 to $Y$ at this point, i.e. it lies in $\bar{M}_{(4,2,2,2)}$. However, this space is empty by Lemma 2.1.

Case 2: the image of the stable map is a union of two (distinct) lines. It is easy to see that the conditions of Remark 1.5 cannot be satisfied in this case.

Case 3: the stable map is a double cover of a line. There are six possible points of tangency to $Y$ : the four inverse image points of the bitangency points, and the two ramification points if they are mapped to points of $Y$. For the stable map in $\bar{M}_{(2,2,2,2,2)}$ we can pick any five of these six points. If we leave out one of the points over the bitangency points, we arrive at the components (B), otherwise we get the components $(\mathrm{C})$. 


\section{A. Gathmann}

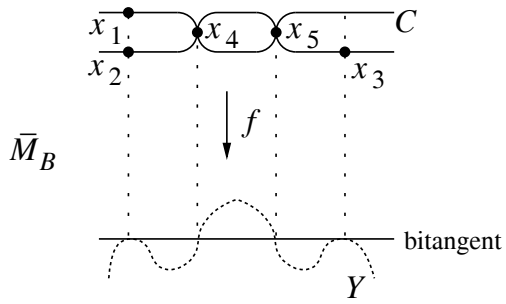

(a)

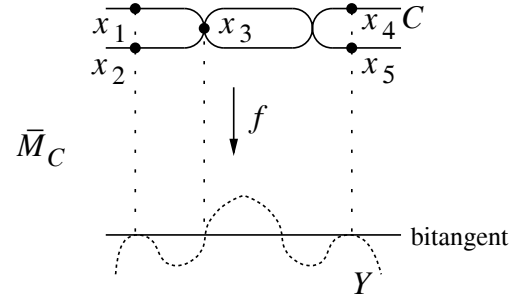

(b)

FiguRE 3.

In case (B) we get a factor of 5 ! for the choice of labeling of the marked points, a factor of $\left(\begin{array}{c}d-4 \\ 2\end{array}\right)$ for the choice of two transverse intersection points of $Y$ with the bitangent, and a factor of two for the choice of bitangency point over which we take only one inverse image point to be marked. In case $(\mathrm{C})$ the second ramification point is not fixed, so we get one-dimensional families of such curves. Every such family has a 2:1 map to the bitangent given by the image of the moving ramification point; the two stable maps in a fiber of this map differ by exchanging the marked points over one bitangency point. The map is simply ramified over the two stable maps where the moving ramification point is one of the bitangency points. Hence, every such family is a $\mathbb{P}^{1}$. The number of such families is $d-4$ (for the choice of transverse intersection point of the bitangent with $Y$ ) times $\frac{1}{4} \cdot 5$ ! (for the labeling of the marked points, taking into account that exchanging the marked points over the bitangency points does not give us a new family).

Of course, the virtual fundamental class of $\bar{M}_{(2,2,2,2,2)}$ splits naturally into a sum of virtual fundamental classes on each of the connected components that we have just identified. As it is well known that the number of bitangents of $Y$ is $\frac{1}{2} d(d+3)(d-2)(d-3)$ (see, e.g., [Har77, Exercise IV.2.3f]), we get the following corollary.

Corollary 2.5. We have

$$
N_{d}=n_{d}+\frac{1}{2} d(d+3)(d-2)(d-3)(d-4)(d-5) b_{d}+\frac{1}{8} d(d+3)(d-2)(d-3)(d-4) c_{d},
$$

where $b_{d}$ is the degree of the part of the virtual fundamental class of $\bar{M}_{(2,2,2,2,2)}$ supported on the point $\bar{M}_{B} \in \bar{M}_{(2,2,2,2,2)}$ shown in Figure 3, and $c_{d}$ is the corresponding degree supported on the smooth rational curve $\bar{M}_{C} \subset \bar{M}_{(2,2,2,2,2)}$.

\section{Computation of the virtual fundamental classes}

In this section we do the necessary computations to determine the numbers $b_{d}$ and $c_{d}$ of Corollary 2.5. Most of them are simple calculations in local coordinates, so we only sketch these parts and leave the details to the reader.

The computation of $b_{d}$ is quite simple, as the component $\bar{M}_{B}$ of $\bar{M}_{(2,2,2,2,2)}$ has the expected dimension.

LEMma 3.1. We have $b_{d}=1$ for all $d$.

Proof. By Remark 1.4 we just have to show that the 10 equations of vanishing of the 1-jets of $e v_{i}^{*} F$ for $i=1, \ldots, 5$ locally cut out the point $\bar{M}_{B}$ in $\bar{M}_{0,5}\left(\mathbb{P}^{2}, 2\right)$ scheme-theoretically with multiplicity 1 . Let us start with the 1 -jets at the points $x_{1}, x_{2}, x_{3}$, i.e. with the space $\bar{M}_{(2,2,2)}$. We can choose the coordinates on $\mathbb{P}^{2}$ such that the bitangent is $\left\{z_{2}=0\right\} \subset \mathbb{P}^{2}$ and the bitangency points are $(1: 0: 0)$ 


\section{The NUMBer of PlANE CONICS THAT ARE FIVE-FOLD TANGENT TO A GIVEN CURVE}

and $(0: 1: 0)$. This means that

$$
\begin{aligned}
& a_{(d, 0,0)}=a_{(d-1,1,0)}=0, \quad a_{(d-1,0,1)}, a_{(d-2,2,0)} \neq 0 \quad(\text { tangency at }(1: 0: 0)), \\
& \left.a_{(0, d, 0)}=a_{(1, d-1,0)}=0, \quad a_{(0, d-1,1)}, a_{(2, d-2,0)} \neq 0 \quad \text { (tangency at }(0: 1: 0)\right) .
\end{aligned}
$$

Moreover, we can choose coordinates on the source $\mathbb{P}^{1}$ such that the stable map $\bar{M}_{B}$ is given by $(s: t) \mapsto\left(s^{2}-t^{2}: s t: 0\right)$, and the marked points are $x_{1}=(1: 0), x_{2}=(0: 1), x_{3}=(1: 1)$. Local coordinates of $\bar{M}_{0,3}\left(\mathbb{P}^{2}, 2\right)$ around this point are then $\epsilon_{1}, \ldots, \epsilon_{8}$, where the stable map is given by

$$
(s: t) \mapsto\left(s^{2}-t^{2}+\epsilon_{1} s^{2}+\epsilon_{2} s t+\epsilon_{3} t^{2}: s t+\epsilon_{4} s^{2}+\epsilon_{5} t^{2}: \epsilon_{6} s^{2}+\epsilon_{7} s t+\epsilon_{8} t^{2}\right) .
$$

The three tangency equations are that $\left.F\right|_{s=1, t=\xi},\left.F\right|_{s=\xi, t=1}$, and $\left.F\right|_{s=1, t=1+\xi}$ have neither constant nor linear $\xi$ terms. It is an easy computation to see that these six equations, linearized in the $\epsilon_{i}$, give

$$
\epsilon_{1}+\epsilon_{2}+\epsilon_{3}=\epsilon_{4}=\epsilon_{5}=\epsilon_{6}=\epsilon_{7}=\epsilon_{8}=0,
$$

so $\bar{M}_{(2,2,2)}$ is smooth of dimension 2 at the point $\bar{M}_{B}$ (with the points $x_{4}$ and $x_{5}$ forgotten).

Now let us consider the two other tangency conditions at the points $x_{4}$ and $x_{5}$. As the four coordinates of $\bar{M}_{(2,2,2,0,0)}$ around $\bar{M}_{B}$ we can choose the images of the ramification points and a point in the domain of the stable map in the neighborhood of each ramification point. Considering only one ramification point for now, the two corresponding local coordinates are $\tilde{\epsilon}_{1}$ and $\tilde{\epsilon}_{2}$, where the stable map is given locally in affine coordinates as $t \mapsto t^{2}+\tilde{\epsilon}_{1}$, and the marked point is $t=\tilde{\epsilon}_{2}$. Tangency means that the constant and linear $\xi$ terms of $\left(\tilde{\epsilon}_{2}+\xi\right)^{2}+\tilde{\epsilon}_{1}$ vanish, so linearly in $\tilde{\epsilon}_{i}$ we get $\tilde{\epsilon}_{1}=\tilde{\epsilon}_{2}=0$. The same is true for the other ramification point, so we see that $\bar{M}_{B}$ is a smooth point of $\bar{M}_{(2,2,2,2,2)}$.

To study the space $\bar{M}_{C}$, we need a lemma that tells us how the stable maps in $\bar{M}_{C}$ can be deformed if we relax some of the multiplicity conditions.

Lemma 3.2. Let $H$ be a line in $\mathbb{P}^{2}$, and let $P \in H$ be a point where $Y$ is simply tangent to $H$. Let $\mathcal{C}=$ $\left(C, x_{1}, x_{2}, f\right) \in \bar{M}_{(2,2)}$ be a (possibly reducible) double cover of $H$, such that $f^{-1}(P)=\left\{x_{1}, x_{2}\right\}$. Then every stable map in $\bar{M}_{(2,2)}$ in a neighborhood of $\mathcal{C}$ is also a double cover of a (maybe different) line.

Proof. It is obvious that $\mathcal{C}$ cannot be deformed into a union of two distinct lines in $\bar{M}_{(2,2)}$. So we have to show that $\mathcal{C}$ cannot be deformed to an irreducible smooth conic in $\bar{M}_{(2,2)}$.

We use the classical space of complete conics (which is isomorphic to $\bar{M}_{0,0}\left(\mathbb{P}^{2}, 2\right)$ ). Recall that this space is the closure in $\mathbb{P}^{5} \times\left(\mathbb{P}^{5}\right)^{\vee}$ of the set $\left(C, C^{\vee}\right)$, where $C$ is an irreducible conic and $C^{\vee}$ its dual. For our given point $\mathcal{C}$ (with the two marked points forgotten for the moment), $C$ is the double line $H$, and $C^{\vee}$ is the union of the two lines in $\mathbb{P}^{\vee}$ that correspond to the two ramification points of $f$ in $\mathbb{P}^{2}$. Assume that we can deform $\left(C, C^{\vee}\right)$ in the space of complete conics to an irreducible conic that is still tangent to $Y$ at two points in the neighborhood of $P$. In particular, we would then deform $C^{\vee}$ to an irreducible conic that is tangent to the dual of $Y$ (or, more precisely, if $H$ is a bitangent of $Y$ : to the branch of the dual of $Y$ that corresponds to the point $P$ ) at two points in this neighborhood. By the continuity of intersection products this means that both lines of $C^{\vee}$ must actually be the line corresponding to the point $P$. Hence, both ramification points of $f$ would have to be $P$. This means that $\mathcal{C}$ must have a contracted rational component over $P$, in contradiction to the assumption $f^{-1}(P)=\left\{x_{1}, x_{2}\right\}$.

We want to reduce the computation of $c_{d}$ to spaces that have the expected dimension. To do this, we use Equation (2) from Example 1.7. Note that by Remark 1.12 this equation is true in the Chow group of the geometric zero locus of the section $\sigma$ (that describes the tangency condition and whose 


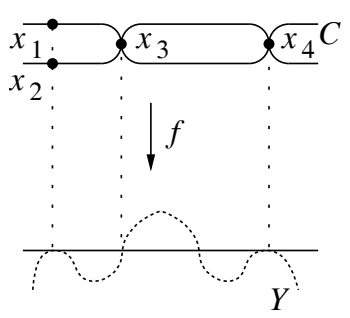

(a)

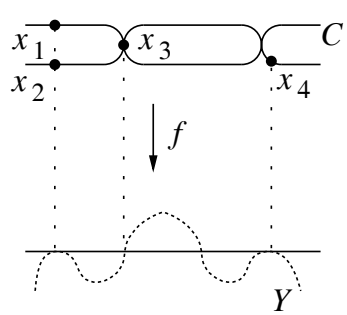

(b)

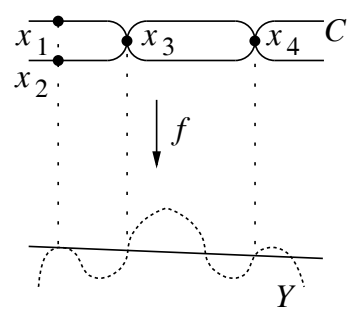

(c)

FIGURE 4.

zero locus has class $e v_{5}^{*} Y+\psi_{5}$ ), so it makes sense to restrict the equation to a connected component of this zero locus. Using Lemma 3.2 it is easy to see that $\bar{M}_{C}$ is such a connected component, so we restrict Equation (2) to $\bar{M}_{C}$ and denote this restriction by $\left.\right|_{\bar{M}_{C}}$.

Note first that on $\bar{M}_{C}$ the point $x_{5}$ can only come close to $x_{4}$, but never to the other three marked points. Hence, Equation (2) restricted to $\bar{M}_{C}$ reads

$$
\left.\left(\left(e v_{5}^{*} Y+\psi_{5}\right) \cdot\left[\bar{M}_{(2,2,2,2,1)}\right]\right)\right|_{\bar{M}_{C}}=c_{d}+\left.3\left[\bar{M}_{(2,2,2,3)}\right]\right|_{\bar{M}_{C}} .
$$

Let us first compute the virtual fundamental classes occurring in this equation.

Lemma 3.3. We have the following.

(i) The (one-dimensional) virtual fundamental class of $\bar{M}_{(2,2,2,2,1)}$ on $\bar{M}_{C}$ is twice the usual one.

(ii) The degree of the (zero-dimensional) virtual fundamental class of $\bar{M}_{(2,2,2,3)}$ on $\bar{M}_{C}$ is 3 .

Proof. We only sketch the computations.

(i) It is enough to do the computation at a general point of $\bar{M}_{C}$. We have seen in the proof of Lemma 3.1 that $\bar{M}_{(2,2,2,2,0)}$ is smooth of dimension 2 at a general point of $\bar{M}_{C}$. However, requiring multiplicity 1 at the point $x_{5}$ gives us a factor of two (i.e. $e v_{5}^{*} Y$ cuts out $\bar{M}_{C}$ in $\bar{M}_{(2,2,2,2,0)}$ with multiplicity 2) because $x_{5}$ lies on a tangency point of the stable map with $Y$.

(ii) The only point of $\bar{M}_{(2,2,2,3)}$ in $\bar{M}_{C}$ is the stable map in Figure 4(a). Its multiplicity can be computed using Remark 1.4. Let us study the space $\bar{M}_{(2,2,2,2)}$ at this point first. By Lemma 3.2 every stable map in $\bar{M}_{(2,2,2,2)}$ in a neighborhood of this point must also be a double cover of a line. It follows easily that, locally around this point, $\bar{M}_{(2,2,2,2)}$ is reducible, with two smooth onedimensional components coming together: one of them is keeping the image line $f(C)$ to be the bitangent and moving the ramification point at $x_{4}$, while keeping $x_{4}$ on an inverse image point of the bitangency point (Figure 4(b)). A local coordinate for this component is $\epsilon$, where the stable map is given locally in affine coordinates as $t \mapsto z=t(t+\epsilon)$, and the marked point $x_{4}$ has coordinate $t=0$. Now the equation $F$ of $Y$ vanishes on the bitangent with multiplicity 2 in $z$, so this equation pulled back to the curve is locally $t^{2}(t+\epsilon)^{2}$. Its $t^{2}$ coefficient vanishes to order 2 in $\epsilon$, so this component contributes 2 to the virtual fundamental class of $\bar{M}_{(2,2,2,3)}$. The other component is deforming the line in $\mathbb{P}^{2}$ away from the bitangent, with marked points as in Figure 4(c). Requiring multiplicity 3 at $x_{4}$ now restricts the line back to the bitangent with multiplicity 1 . Hence, the total degree of the virtual fundamental class of $\bar{M}_{(2,2,2,3)}$ on $\bar{M}_{C}$ is $2+1=3$.

To evaluate the left-hand side of Equation (3) it is not enough to compute the integral of $e v_{5}^{*} Y+$ $\psi_{5}$ on $\bar{M}_{C}$. There may also be contributions from components of $\bar{M}_{(2,2,2,2,1)}$ that just intersect $\bar{M}_{C}$, if the section $\sigma$ above (whose zero locus has class $e v_{5}^{*}+\psi_{5}$ ) vanishes on them at a point of $\bar{M}_{C}$. Let us compute these contributions.

Lemma 3.4. The only component $Z$ of $\bar{M}_{(2,2,2,2,1)}$ that meets $\bar{M}_{C}$, but is not $\bar{M}_{C}$ itself, corresponds to double covers of simple tangent lines of $Y$, with ramification and marked points as in Figure 5(a). 


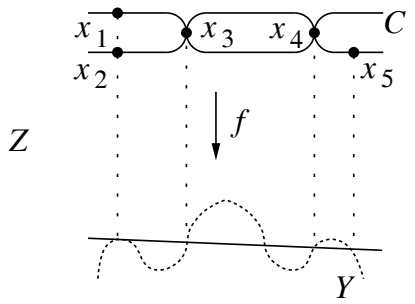

(a)

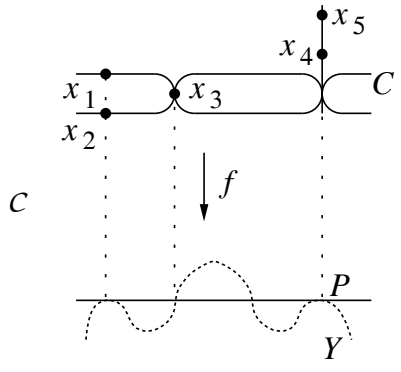

(b)

FiguRE 5.

Its virtual fundamental class is equal to the usual one. It intersects $\bar{M}_{C}$ in the point $\mathcal{C}$ in Figure $5(b)$. The section $\sigma$ on $Z$ vanishes at $\mathcal{C}$ with multiplicity 4 .

Proof. By Lemma 3.2 (applied to the two marked points $x_{1}$ and $x_{2}$ ) no stable map in $\bar{M}_{C}$ can be deformed into an element in $\bar{M}_{(2,2)}$ that is not itself a double cover of a line. So the only possible deformation is that we still have a double cover, however not of the bitangent but rather a nearby line. It is now easy to see that such a deformation is only possible for the stable map $\mathcal{C} \in \bar{M}_{C}$ in Figure 5(b), and that the deformation has to be that in Figure 5(a).

The computation of the virtual fundamental class on this component $Z$ is completely analogous to similar calculations in previous lemmas and is therefore omitted. It remains to compute the order of vanishing of the section $\sigma$ on $Z$ at $\mathcal{C}$. Let us forget for a moment that the stable map splits off a contracted rational component at this point. Let $P=f\left(x_{4}\right)=f\left(x_{5}\right)$ be the bitangency point of $\mathcal{C}$. Choose local affine coordinates $z_{1}, z_{2}$ of $\mathbb{P}^{2}$ around $P$ such that the local equation of $Y$ is $z_{2}=z_{1}^{2}+O\left(z_{1}^{3}\right)$, and $z_{2}=0$ is the bitangent. As a local coordinate for $Z$ around $\mathcal{C}$ we can choose $\epsilon$, where the stable map is given locally around $P$ by $t \mapsto\left(z_{1}=t^{2}-\frac{1}{2} \epsilon^{2}, z_{2}=\frac{1}{4} \epsilon^{4}+O\left(\epsilon^{5}\right)+t^{2} O\left(\epsilon^{4}\right)\right)$, and the marked points $x_{4}$ and $x_{5}$ are $t=0$ and $t=\epsilon$. (Note that this stable map is still a double cover of a line, $t=0$ is a ramification point that maps to $Y$, and $t=\epsilon$ is another point that maps to $Y$.)

Now the stable map actually splits off a rational contracted component at $\mathcal{C}$, which corresponds to blowing up the point $(\epsilon=0, t=0)$ in our family of stable maps. Hence, the true local coordinates of this family of stable maps are the coordinates of this blow-up, i.e. $t / \epsilon$ and $\epsilon$ instead of $t$ and $\epsilon$. So the marked points $x_{4}$ and $x_{5}$ have coordinates $t=0$ and $t / \epsilon=1$; in particular, they no longer coincide at $\epsilon=0$.

The vanishing of the section $\sigma$ is the condition of tangency of $f$ to $Y$ at $x_{5}$. So to compute its order of vanishing at $\mathcal{C}$ we have to look at the linear $\xi$ coefficient of the equation of $Y$ evaluated at the point $t / \epsilon=1+\xi$, i.e. of

$$
z_{2}-z_{1}^{2}+O\left(z_{1}^{3}\right)=\frac{1}{4} \epsilon^{4}-\left(\frac{1}{2} \epsilon^{2}+\xi \epsilon^{2}\right)^{2}+O\left(\epsilon^{5}\right)=-\xi \epsilon^{4}-\xi^{2} \epsilon^{4}+O\left(\epsilon^{5}\right) .
$$

Hence, the linear $\xi$ coefficient vanishes with multiplicity 4 at $\epsilon=0$, which proves the lemma.

We can now assemble the results of our local calculations to compute the number $c_{d}$.

Lemma 3.5. We have $c_{d}=-1$ for all $d$.

Proof. We evaluate Equation (3)

$$
\left.\left(\left(e v_{5}^{*} Y+\psi_{5}\right) \cdot\left[\bar{M}_{(2,2,2,2,1)}\right]\right)\right|_{\bar{M}_{C}}=c_{d}+\left.3\left[\bar{M}_{(2,2,2,3)}\right]\right|_{\bar{M}_{C}} .
$$

The right-hand side is $c_{d}+9$ by Lemma 3.3(ii). The left-hand side gets a contribution from the components of $\bar{M}_{(2,2,2,2,1)}$ that intersect $\bar{M}_{C}$, and a contribution from $\bar{M}_{C} \subset \bar{M}_{(2,2,2,2,1)}$ itself. 


\section{A. Gathmann}

The former is 4 by Lemma 3.4. The latter is twice the degree of $e v_{5}^{*} Y+\psi_{5}$ on $\bar{M}_{C}$ by Lemma 3.3(i). Note that the degree of $e v_{5}^{*} Y$ on $\bar{M}_{C}$ is zero, as the image point of $x_{5}$ is fixed in $\bar{M}_{C}$.

To compute the integral of $\psi_{5}$ on $\bar{M}_{C}$, we give a section of the cotangent line bundle $L_{5}$ and compute its zero locus. Let $z$ be a local coordinate around the bitangency point $f\left(x_{4}\right)=f\left(x_{5}\right)$. Then $f^{*} d z$ defines a section of $L_{5}$. This section vanishes only at the point where the moving ramification point comes to $x_{4}$ and $x_{5}$, i.e. at point $\mathcal{C}$ in Figure 5(b) in Lemma 3.4. The computation of the order of vanishing is very similar to the calculation in Lemma 3.4. Ignoring the fact that $\mathcal{C}$ splits off a rational contracted component for $x_{4}$ and $x_{5}$, a local coordinate for $\bar{M}_{C}$ around $\mathcal{C}$ is $\epsilon$, where the stable map is given locally by $t \mapsto z=t(t-\epsilon)$, the points $x_{4}$ and $x_{5}$ are $t=0$ and $t=\epsilon$, and the moving ramification point is at $t=\frac{1}{2} \epsilon$. Now, as in the proof of the previous lemma, taking into account the contracted rational component of $\mathcal{C}$ means that we have to blow up the point $(t=0, \epsilon=0)$, and the coordinates are actually $t / \epsilon$ and $\epsilon$. Now we see that

$$
f^{*} d z=\frac{\partial}{\partial \frac{t}{\epsilon}}\left(\epsilon^{2} \cdot \frac{t}{\epsilon}\left(\frac{t}{\epsilon}-1\right)\right) d \frac{t}{\epsilon}=\epsilon^{2}\left(2 \frac{t}{\epsilon}-1\right) d \frac{t}{\epsilon},
$$

which vanishes with multiplicity 2 in $\epsilon$ around 0 at the point $x_{5}$. Hence, the integral of $\psi_{5}$ over $\bar{M}_{C}$ is 2 .

Putting everything together, we get $4+2 \cdot 2=c_{d}+9$, and therefore $c_{d}=-1$.

We can now insert the values for $N_{d}, b_{d}$, and $c_{d}$ from Proposition 1.10, Lemma 3.1, and Lemma 3.5, respectively, into the equation from Lemma 2.5, and get the following final result.

Corollary 3.6. For $d \geqslant 5$, the enumerative number of conics five-fold tangent to $Y$ is

$$
n_{d}=\frac{1}{5 !} d(d-3)(d-4)\left(d^{7}+12 d^{6}-18 d^{5}-540 d^{4}+251 d^{3}+5712 d^{2}-1458 d-14580\right) .
$$

Remark 3.7. The first few values of $n_{d}$ are given in the following table.

\begin{tabular}{|c|c|c|c|c|c|c|}
\hline$d$ & 5 & 6 & 7 & 8 & 9 & 10 \\
\hline$n_{d}$ & 2015 & 70956 & 684222 & 3878736 & 16137873 & 54575640 \\
\hline
\end{tabular}

\section{Application to rational curves on K3 surfaces}

Let $X$ be a $\mathrm{K} 3$ surface, and let $\beta \subset H_{2}(X, \mathbb{Z})$ be the class of a holomorphic curve in $X$. The moduli space of stable maps to $X$ of class $\beta$ has virtual dimension -1 , so there is no corresponding Gromov-Witten invariant of $X$. However, we have chosen $X$ such that it contains rational curves of class $\beta$, and we would like to count them. The reason for the mismatch in the virtual dimension is that, in the space of all K3 surfaces, only a one-codimensional subset of K3 surfaces contains curves in the class $\beta$ at all. So, if $\mathcal{X}$ is a general one-dimensional family of K3 surfaces with $X$ as central fiber, and $\tilde{\beta} \in H_{2}(\mathcal{X}, \mathbb{Z})$ is the class induced by $\beta$ via the inclusion $X \subset \mathcal{X}$, then the only rational curves in $\mathcal{X}$ of class $\tilde{\beta}$ are in fact curves of class $\beta$ in $X$. However, now the virtual dimension of the space of stable maps to $\mathcal{X}$ of class $\beta$ is zero, hence there is a corresponding Gromov-Witten invariant. This invariant counts curves in $\mathcal{X}$ of class $\tilde{\beta}$ and therefore curves in $X$ of class $\beta$; so we would like to call this number $n_{\beta}$ 'the number of rational curves in $X$ of class $\beta$ '. A rigorous definition of the invariant $n_{\beta}$ of $X$ along these lines has been given in [BL00]. The number $n_{\beta}$ does not depend on the family $\mathcal{X}$ chosen to define it.

The numbers $n_{\beta}$ have been computed in various papers ([YZ96, Bea99, Got98, BL00]) under the assumption that the class $\beta$ is primitive, i.e. not a non-trivial multiple of a smaller integral 


\section{The NUMBer of PlANE CONICS THAT ARE FIVE-FOLD TANGENT TO A GIVEN CURVE}

homology class. The result is that $n_{\beta}$ is equal to the $q^{d}$ coefficient in the series

$$
\begin{aligned}
G(q) & =\prod_{i>0} \frac{1}{\left(1-q^{i}\right)^{24}}=1+24 q+324 q^{2}+3200 q^{3}+25650 q^{4}+176256 q^{5}+\cdots \\
& =: \sum_{d \geqslant 0} G_{d} q^{d} .
\end{aligned}
$$

where $d=\frac{1}{2} \beta^{2}+1$. It is not yet known what the numbers are if $\beta$ is not primitive.

The results of this paper allow us to compute the number $n_{\beta}$ explicitly in a case where $\beta$ is not primitive. Let $Y \subset \mathbb{P}^{2}$ be a general sextic curve, and let $\pi: X \rightarrow \mathbb{P}^{2}$ be the double cover of $\mathbb{P}^{2}$ branched along $Y$. It is well-known that $X$ is a K3 surface. Let us start by considering curves on $X$ in the (primitive) class $\beta=\pi^{*} \ell$, where $\ell$ is the class of a line. The pull-back of a general line in $\mathbb{P}^{2}$ will be a 2:1 cover of $\mathbb{P}^{1}$, branched along the six intersection points of $Y$ with the line, hence it is a curve of genus 2. We get a rational curve (with two nodes) on $Y$ as a pull-back of a line if and only if the line is a bitangent of $Y$ : the pull-back is then a 2:1 cover again, but with two nodes (the bitangency points), and only two ramification points (the remaining two intersection points of $Y$ with the line). So we see that $n_{\pi^{*} \ell}$ has to be the number of bitangents of $Y$. In fact, this number is 324 (see, e.g., [Har77, Exercise IV.2.3f]), which is equal to $G_{2}$ (note that $\frac{1}{2}\left(\pi^{*} \ell\right)^{2}+1=2$ ).

Now, let us consider rational curves in $X$ of class $2 \pi^{*} \ell$, i.e. pull-backs of conics: this class is no longer primitive. The pull-back of a general conic will be a 2:1 cover of the conic ramified at 12 points, so it is a curve of genus 5 . We can get rational curves in the following ways.

(i) Pull-backs of (smooth) conics that are five-fold tangent to $Y$. These will be 2:1 covers of the conic, with five nodes over the tangency points, and only two ramification points (the remaining two intersection points of $Y$ with the conic). By Corollary 3.6, there are 70956 such curves.

(ii) Pull-backs of unions of two distinct lines: these give a rational curve only if the pull-backs of both lines are rational, i.e. they are both bitangents. The pull-back is then a union of two (two-nodal) rational curves on $X$ that intersect in two points. To make this into a rational stable map we can glue these two components at either intersection point. Hence, there are $2 \cdot\left(\begin{array}{c}324 \\ 2\end{array}\right)=104652$ such stable maps.

(iii) Double covers of pull-backs of a line, necessarily again of a bitangent. The pull-back of such a bitangent is a two-nodal rational curve $C$. There are two possible ways of double covers of such a curve.

(a) Double covers that factor through the normalization of $C$. The space of these curves is the same as that of double covers of a smooth rational curve; it has dimension 2.

(b) Double covers that do not factor through the normalization. They have two components that are both mapped to $C$ with degree 1, and glued over one of the nodes of $C$ in such a way that, locally around this node, the morphism of the stable map is an isomorphism onto $C$. There are $2 \times 324=648$ such curves.

Adding up just the numbers from (i), (ii), and (iii)(b), we get

$$
70956+104652+648=176256
$$

which is exactly $G_{5}$ (and $\frac{1}{2}\left(2 \pi^{*} \ell\right)+1=5$ ). So we see that, for our non-primitive class $\beta$, the corresponding invariant from the series $G(q)$ does give the correct number, except for a correction (iii)(a) for double covers of curves of class $\frac{1}{2} \beta$ that factor through the normalization of these curves. Let us compute what this correction term is.

Lemma 4.1. With notations as above, the double covers of type (iii)(a) of the pull-back of a bitangent contribute $\frac{1}{8}$ to the invariant $n_{2 \pi^{*} \ell}$. 


\section{A. Gathmann}

Proof. Let $D \cong \mathbb{P}^{1}$ be the normalization of the nodal rational curve in $X$. The moduli space of the double covers that factor through the normalization is then just $\bar{M}_{0,0}(D, 2)$, which has dimension 2 . As the normal bundle of the (local) immersion $D \rightarrow X$ is $\mathcal{O}(-2)$, the rank-3 obstruction bundle for the corresponding Gromov-Witten invariant would be $R^{1} \pi_{*} f^{*} \mathcal{O}(-2)$, where $\pi: \bar{M}_{0,1}\left(\mathbb{P}^{1}, 2\right) \rightarrow$ $\bar{M}_{0,0}\left(\mathbb{P}^{1}, 2\right)$ is the forgetful map and $f: \bar{M}_{0,1}\left(\mathbb{P}^{1}, 2\right) \rightarrow \mathbb{P}^{1}$ the evaluation.

As explained above, the K3-invariants of $X$ are defined as the ordinary Gromov-Witten invariants of a one-dimensional family $\mathcal{X}$ of $\mathrm{K} 3$ surfaces in which $X$ is the only surface that contains rational curves in the given homology class. This means that the obstruction bundle for the K3 invariants is obtained from the usual Gromov-Witten obstruction bundle by taking the quotient with $\pi_{*} f^{*} N_{X / \mathcal{X}}=\pi_{*} f^{*} \mathcal{O}=\mathcal{O}$. So the integral that we want to compute is

$$
c_{\text {top }}\left(R^{1} \pi_{*} f^{*} \mathcal{O}(-2) / \mathcal{O}\right) \cdot\left[\bar{M}_{0,0}\left(\mathbb{P}^{1}, 2\right)\right] .
$$

This is easily done: from the two exact sequences on $\bar{M}_{0,1}\left(\mathbb{P}^{2}, 2\right)$

$$
\begin{gathered}
0 \rightarrow f^{*} \mathcal{O}(-1) \rightarrow f^{*} \mathcal{O} \rightarrow f^{*} \mathcal{O}_{P} \rightarrow 0 \\
0 \rightarrow f^{*} \mathcal{O}(-2) \rightarrow f^{*} \mathcal{O}(-1) \rightarrow f^{*} \mathcal{O}_{P} \rightarrow 0
\end{gathered}
$$

(where $P \in \mathbb{P}^{1}$ is a point) we get the exact sequences of vector bundles on $\bar{M}_{0,0}\left(\mathbb{P}^{2}, 2\right)$

$$
\begin{aligned}
0 & \rightarrow \mathcal{O} \rightarrow \pi_{*} f^{*} \mathcal{O}_{P} \rightarrow R^{1} \pi_{*} f^{*} \mathcal{O}(-1) \rightarrow 0 \\
0 \rightarrow \pi_{*} f^{*} \mathcal{O}_{P} & \rightarrow R^{1} \pi_{*} f^{*} \mathcal{O}(-2) \rightarrow R^{1} \pi_{*} f^{*} \mathcal{O}(-1) \rightarrow 0
\end{aligned}
$$

from which it follows that

$$
c_{2}\left(R^{1} \pi_{*} f^{*} \mathcal{O}(-2) / \mathcal{O}\right)=c_{2}\left(R^{1} \pi_{*} f^{*}(\mathcal{O}(-1) \oplus \mathcal{O}(-1))\right),
$$

i.e. the contribution of the double covers under consideration is the same as the double cover contribution for rational curves on Calabi-Yau threefolds with balanced normal bundle. This contribution is well known to be $\frac{1}{8}$; see, e.g., [Gat01, Example 6.3].

So we see that

$$
n_{2 \pi^{*} \ell}=G_{5}+\frac{1}{8} \cdot G_{2} .
$$

We conjecture that this pattern continues, i.e. that the numbers $n_{\beta}$ receive multiple cover corrections similarly to the case of Gromov-Witten invariants of Calabi-Yau threefolds:

$$
n_{\beta}=\sum_{k} \frac{1}{k^{3}} \cdot G_{1 / 2(\beta / k)^{2}+1}
$$

where the sum is taken over all $k>0$ such that $\beta / k$ is an integral homology class.

\section{ACKNOWLEDGEMENTS}

We thank J. Kock for introducing us to the problem, and also for a Maple program used to compute the relative Gromov-Witten invariants using the algorithm of [Gat02]. The author is also grateful to the Institute for Advanced Study for its hospitality and stimulating working atmosphere.

\section{REFERENCES}

Bea99 A. Beauville, Counting rational curves on K3 surfaces, Duke Math. J. 97 (1999), 99-108.

BL00 J. Bryan and N. Leung, The enumerative geometry of K3 surfaces and modular forms, J. Amer. Math. Soc. 13 (2000), 371-410.

Gat01 A. Gathmann, Gromov-Witten invariants of blow-ups, J. Algebraic Geom. 10 (2001), 399-432. 
The number of Plane Conics that ARe Five-Fold tangent to A Given CuRve

Gat02 A. Gathmann, Absolute and relative Gromov-Witten invariants of very ample hypersurfaces, Duke Math. J. 115 (2002), 171-203.

Got98 L. Göttsche, A conjectural generating function for numbers of curves on surfaces, Comm. Math. Phys. 196 (1998), 523-533.

Har77 R. Hartshorne, Algebraic geometry, Graduate Texts in Mathematics, vol. 52 (Springer, New YorkHeidelberg, 1977).

Vai98 I. Vainsencher, Conics five-fold tangent to a plane curve, Mat. Contemp. 14 (1998), 201-214.

YZ96 S. Yau and E. Zaslow, BPS states, string duality, and nodal curves on K3, Nucl. Phys. B 471 (1996), 503-512.

Andreas Gathmann andreas@mathematik.uni-kl.de

Fachbereich Mathematik, Technische Universität Kaiserslautern, Postfach 3049,

67653 Kaiserslautern, Germany 